\title{
LOWER BOUND YIELD LOCUS CALCULATIONS
}

\author{
WILLIAM HOSFORD $\dagger$ and AITOR GALDOS $\ddagger$ \\ University of Michigan, Department of Materials Science and Engineering, \\ Ann Arbor, Michigan, 48109 USA
}

(Received February 15, 1989)

\begin{abstract}
A lower-bound model for the deformation of work-hardening polycrystals is proposed. All grains are assumed to be loaded under the same stress and the stress-strain behavior is found by averaging the strains in all grains. The shapes of the yield loci have been calculated for textured metals which deform by $\{111\}\langle 110\rangle$ slip (fcc) and by $\langle 111\rangle$-pencil glide (bcc). As with the corresponding upper-bound models, the yield loci are best described by an anisotropic yield criterion with an exponent of 6 to 10 (instead of 2 as in the Hill theory). Also it is shown that a model of polycrystal deformation in which the grains are loaded to the same stress ratio (but not the same level of stresses) violates normality and is not a lower bound.
\end{abstract}

KEY WORDS Yield locus, lower bound calculation, work-hardening, textured polycrystal

\section{INTRODUCTION}

Upper- and lower-bound analyses are based on well established principles (Drucker et al. 1951, Hill 1951). For a valid upper bound, a geometrically self-consistent internal deformation field must be assumed and the external stresses are calculated from the internal work necessary to produce such deformation. The Taylor/Bishop and Hill model (Taylor 1938, Bishop and Hill 1951) is an upper-bound model in which the geometrically self-consistent deformation field is assured by assuming that every grain undergoes the same shape change. The stress is averaged over all grains. However this model is not exact because it implies different stresses in neighboring grains and hence unbalanced forces acting across grain boundaries. This type of upper-bound model has been used to calculate yield loci for textured polycrystals of fcc metals which deform by $\{111\}\langle 110\rangle$ slip (Hosford 1979, Piehler 1967, Piehler and Backofen 1969) and bcc metals which deform by $\langle 111\rangle$ pencil glide (Piehler 1967, Piehler and Backofen 1969, Semiatin et al. 1979, Logan and Hosford 1980).

Lower-bound analyses are quite different. Here one must assume a selfconsistent internal stress field (one that is statically admissible), but no regard need be taken of strain compatibility. The simplest way to assure self-consistent stresses is to assume that every grain is loaded under exactly the same stress state and that the mean (overall) strain is the average of the strains in the individual grains. Figure 1 illustrates the difference between a lower-bound average and an upper-bound average for a polycrystal containing three grains. The workhardening of each grain is described by a power law, $\sigma=K \varepsilon^{n}$. It is assumed that

Professor $\dagger$ and former graduate student, $\ddagger$ University of Michigan, Ann Arbor MI. 

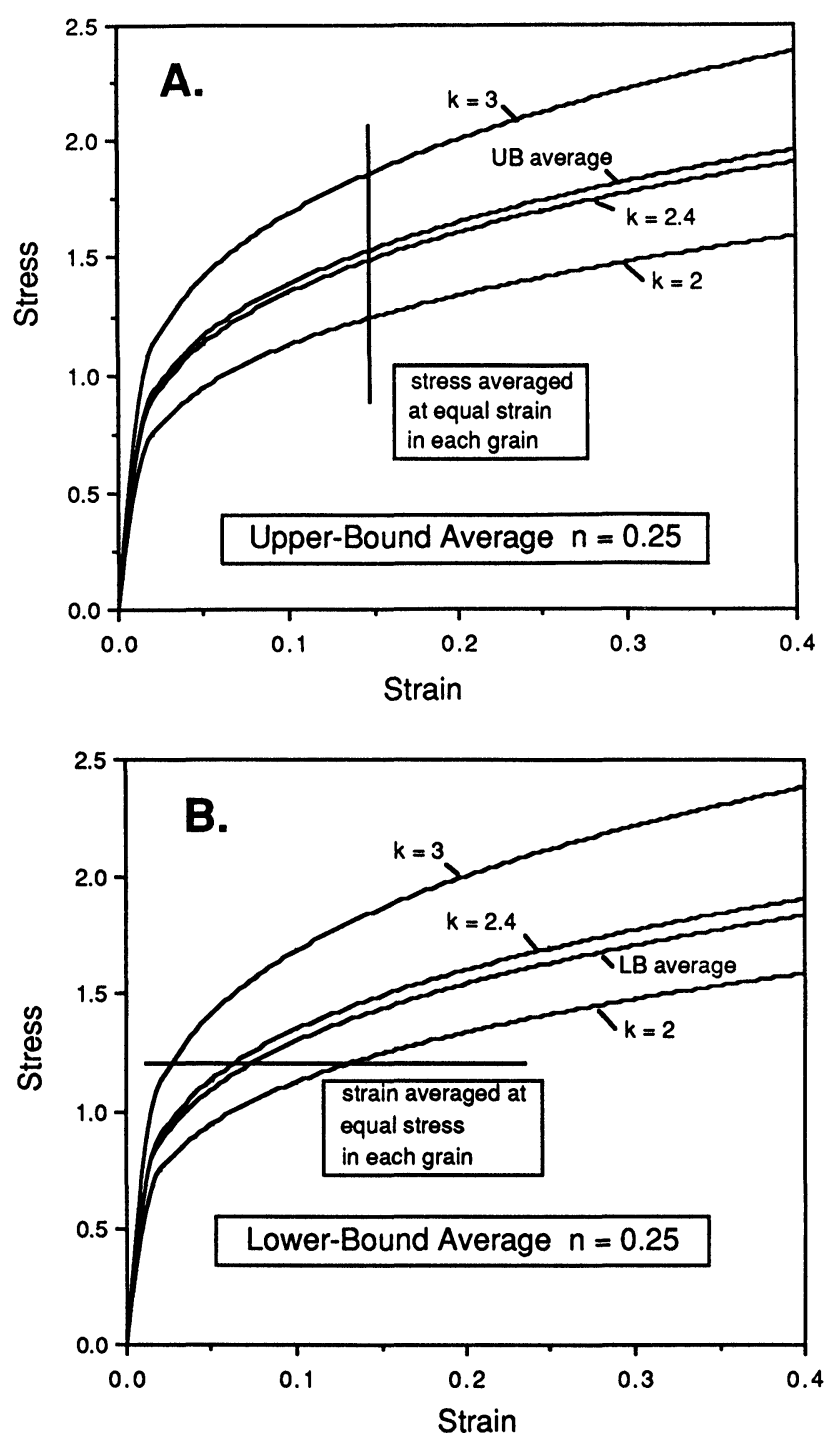

Figure 1 Upper- and lower-bound average stress-strain curves for a polycrystal composed of three grains with $k=2,2.4$ and 3 . For the upper bound (A), the same strain is assumed in each grain and the stresses are averaged at the strain. For the lower bound (B), the same stress is assumed in each grain and the strains are averaged at that stress.

$n=0.25$ for each grain but the value of $k$ varies from grain to grain depending on orientation.

The lower-bound model is reasonable only if work hardening is assumed. Otherwise (if there is no work-hardening) the stress cannot rise above the level that is required for slip in the most favorably oriented (softest) grain, so the calculated strength of the polycrystal is identical to the strength of the softest 


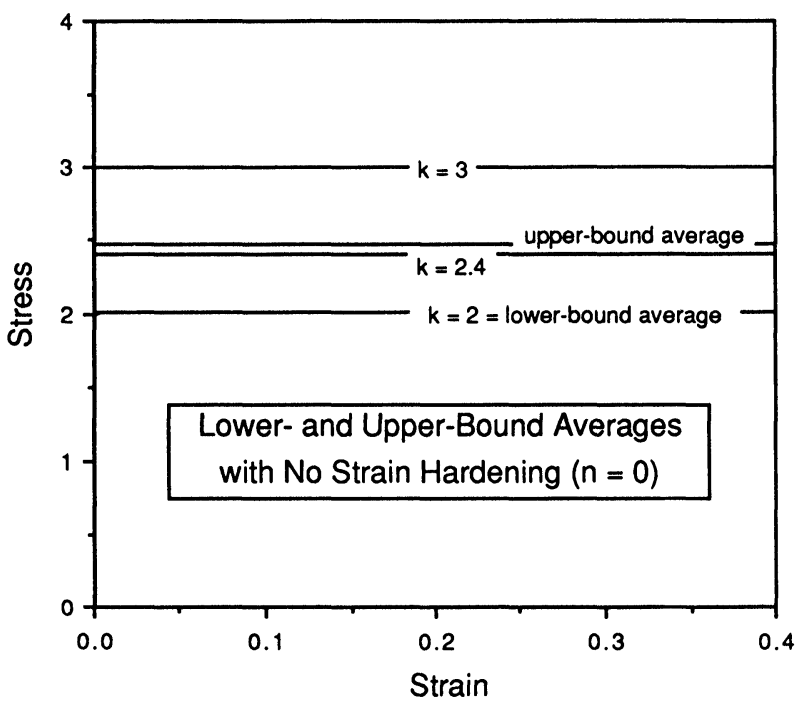

Figure 2 Upper- and lower bound averages with no workhardening. Without workhardening the lower bound model becomes unreasonable, predicting that only the softest grain deforms so the average strength equals the strength of the weakest grain. The upperbound remains reasonable because it averages the stresses.

grain as illustrated for uniaxial tension in Figure 2. Clearly this is unreasonable; the softest grain may deform more than the others, but not infinitely more. Strain hardening can be incorporated into upper-bound Taylor/Bishop \& Hill type calculations (Hosford 1980) but it does not have a large effect on the results because the averaging is done at the same level of strain in each grain so the average stress depends equally on all grains.

\section{CALCULATION METHOD}

In the present calculations power-law work hardening is assumed in each grain with

$$
\tau_{i}=k \gamma_{i}^{n},
$$

where $\tau_{i}$ is the shear stress necessary to cause slip on the active slip system in that grain and $\gamma_{i}$ is the shear strain on the slip system. With this assumption all grains make some contribution to the overall strain and therefore have some effect on the calculated yielding behavior.

Yielding is defined to occur when the plastic work/volume of the polycrystal reaches a critical level, $w$. This is equivalent to defining yielding in terms of an effective strain, $\bar{\varepsilon}$, since $\bar{\sigma}$ and $\bar{\varepsilon}$ are constant everywhere on the yield surface and $w=\int \bar{\sigma} d \bar{\varepsilon}$. This definition of yielding avoids the complication which would arise if yielding were defined as occurring when a critical level of effective strain is reached. The difficulty with that approach is that the appropriate effective stress 
and strain functions are not known until after the yield locus shape has been determined.

The basic assumptions are:

1. A plane-strain stress state, $\sigma_{x}$ and $\sigma_{y}=\alpha \sigma_{x}$ with $\sigma_{z}=0, \tau_{y z}=\tau_{z x}=\tau_{x y}=0$. The coordinate system is shown in Figure 3.

2. No restriction on the resulting shape change, so that slip in each grain occurs only on the system with the highest resolved shear stress.

3. Power-law hardening (Eq. (1))

4. Textures with rotational symmetry about $z$.

In an individual grain, $i$, the plastic work/volume, $w_{i}$, is

$$
w_{i}=\int \tau_{i} d \gamma_{i}=k \gamma_{i}^{n+1} /(n+1)=k^{-1 / n} \tau_{i}^{(n+1) / n} /(n+1),
$$

where $\tau_{i}$ and $\gamma_{i}$ are the shear stress and shear strain on the most heavily stress slip system in each grain. For a polycrystal consisting of $\boldsymbol{g}$ grains of equal size, the overall plastic work per volume, $w$, is simply

$$
w=(1 / g) \sum w_{i}=(q / g) \sum \tau_{i}^{(n+1) / n}
$$

where

$$
q=1 /\left[(n+1) k^{1 / n}\right] .
$$

For a given stress ratio, $\alpha$, the shear stress, $\tau_{i}$ is given by

$$
\tau_{i}=m_{x i} \sigma_{x}+m_{y i} \sigma_{y}=\sigma_{x}\left(m_{x i}+\alpha m_{y i}\right) \text {. }
$$

Here the resolving factor $m_{x i}=\cos \lambda_{x i} \cos \phi_{x i}$ where $\lambda_{x i}$ and $\phi_{x i}$ are the angles between the $x$ direction and, respectively, the slip direction and slip-plane normal of the most heavily stressed slip system. The resolving factor $m_{y i}$ is defined



Figure 3 Loading paths and coordinate system. Rotational symmetry about $z$ and plane-stress $\left(\sigma_{z}=0\right)$ loading are assumed. The four loading paths analyzed are: (1) Uniaxial tension, $\left(\sigma_{y}=\right.$ $0)$; (2) Plane strain $\left(\varepsilon_{y}=0\right)$; (3) biaxial tension $\left(\sigma_{y}=\sigma_{x}, \varepsilon_{y}=\varepsilon_{x}\right)$; and (4) plane strain $\left(\varepsilon_{z}=0\right.$, $\left.\sigma_{y}=-\sigma_{x}\right)$. For uniaxial tension, the resulting ratio of strains, $r=\varepsilon_{y} / \varepsilon_{x}$ was also found. 
similarly. Substitution of Eq. (4) into Eq. (3) gives

$$
w=(q / g) \sum\left[\sigma_{x}\left(m_{x i}+\alpha m_{y i}\right)\right]^{(n+1) / n} \text {. }
$$

Because $\sigma_{x}$ is assumed to be the same in all grains, it can be removed from the summation. Taking $w / q=w k^{1 / n}(n+1)$ as unity and solving for $\sigma_{x}$,

$$
\sigma_{x}=\left[g / \sum\left(m_{x i}+\alpha m_{y i}\right)^{(n+1) / n}\right]^{n /(n+1)}, \quad \text { and } \sigma_{y}=\alpha \sigma_{x} .
$$

The ratio of the resulting strains, $\varepsilon_{y} / \varepsilon_{x}$, can be found in a similar manner. In each grain $\varepsilon_{x i}=m_{x i} \gamma_{i}$ and $\varepsilon_{y i}=m_{y i} \gamma_{i}$ where $\gamma_{i}=\left(\tau_{i} / k\right)^{1 / n}$ and $\tau_{i}=\left(m_{x i}+\alpha m_{y i}\right) \sigma_{x}$. Substituting,

$$
\varepsilon_{x i}=m_{x i}\left[\sigma_{x}\left(m_{x i}+\alpha m_{y i}\right) / k\right]^{1 / n} \text { and } \varepsilon_{y i}=m_{y i}\left[\sigma_{x}\left(m_{x i}+\alpha m_{y i}\right) / k\right]^{1 / n} \text {. }
$$

Therefore the ratio of the external strains, $\varepsilon_{x}=(1 / g) \sum \varepsilon_{x i}$ and $\varepsilon_{y}=(1 / g) \sum \varepsilon_{y i}$, is

$$
\varepsilon_{y} / \varepsilon_{x}=\sum \varepsilon_{y i} / \sum \varepsilon_{x i}=\sum\left[m_{y i}\left(m_{x i}+\alpha m_{y i}\right)^{1 / n}\right] / \sum\left[m_{x i}\left(m_{x i}+\alpha m_{y i}\right)^{1 / n}\right]
$$

Lower-bound calculations were made for both $\{111\}\langle 110\rangle$ slip characteristic of fcc metals and $\langle 111\rangle$-pencil glide characteristics of bcc metals. Both sets of calculations were made assuming strain-hardening exponents of $n=0.1,0.25$ and 0.5. One hundred different textures were analyzed. Each texture consisted of five randomly chosen sheet normals, each sheet normal having 15 different orientations differing from each other by $12^{\circ}$ rotations about the sheet normal. Instead of exploring the entire yield loci for each texture, calculations were made of the yielding behavior along four characteristic loading paths illustrated in Figure 3: (1) uniaxial tension $\left(\sigma_{y}=0\right)$, (2) plane strain $\left(\varepsilon_{y}=0\right)$, (3) biaxial tension $\left(\varepsilon_{y}=\varepsilon_{x}\right.$ and $\left.\sigma_{y}=\sigma_{x}\right)$ and (4) plane strain $\left(\varepsilon_{z}=0, \sigma_{y}=-\sigma_{x}\right)$. For uniaxial tension (path 1) the strain ratio, $R=\varepsilon_{y} / \varepsilon_{z}$, was determined as $R=r /(1-r)$ where $r$ is the value of $-\left(\varepsilon_{y} / \varepsilon_{x}\right)$ in Eq. (8) with $\alpha=0$. For the plane-strain $\left(\varepsilon_{y}=0\right)$, path (2), the corresponding stress ratio, $\alpha^{*}$, was to be found by iteration. Various values of $\alpha$ were assumed until Eq. (8) resulted in $\varepsilon_{y} / \varepsilon_{x}=0$. Three ratios of the yield stresses along the several loading paths, namely

$$
\begin{aligned}
& \beta=\sigma_{x(\text { path } 2)} / 2 \sigma_{x(\text { path } 4)}, \\
& \lambda=\sigma_{x(\text { path } 2)} / \sigma_{x(\text { path } 1)}, \\
& \chi=\sigma_{x(\text { path })} / \sigma_{x(\text { path } 1)} .
\end{aligned}
$$

were calculated together with the $R$-value in uniaxial tension for each of the assumed textures.

The results for the fcc model are given in figures 4 through 6 . Figures $4 \mathrm{a}-\mathrm{c}$ show the calculated ratio, $\beta$, of the yield strengths in plane strain $\left(\varepsilon_{y}=0\right)$ to plane strain $\left(\varepsilon_{z}=0\right)$ as a function of the calculated $R$-value. Each point represents one of the randomly chosen textures. The predictions of the two yield criteria are shown for comparison. The 1948 Hill criterion (Hill 1948, Hill 1950) expressed for planar isotropy and plane-stress loading,

$$
\sigma_{x}^{2}+\sigma_{y}^{2}+R\left(\sigma_{x}-\sigma_{y}\right)^{2}=(R+1) Y^{2}
$$

predicts

$$
\beta=(R+1) /(2 R+1)^{1 / 2} .
$$




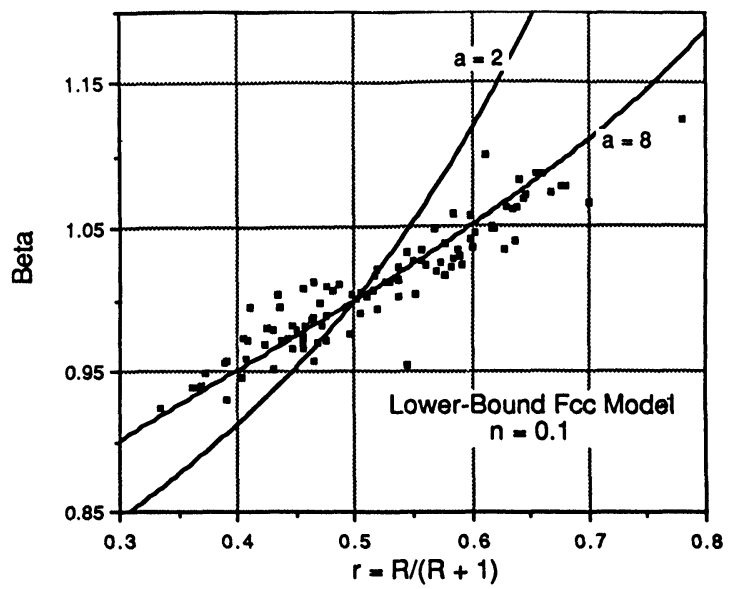

A.

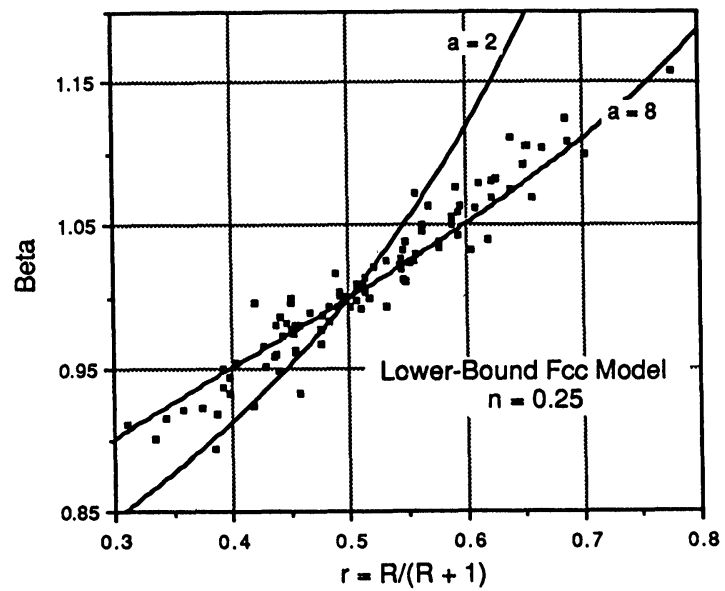

B.

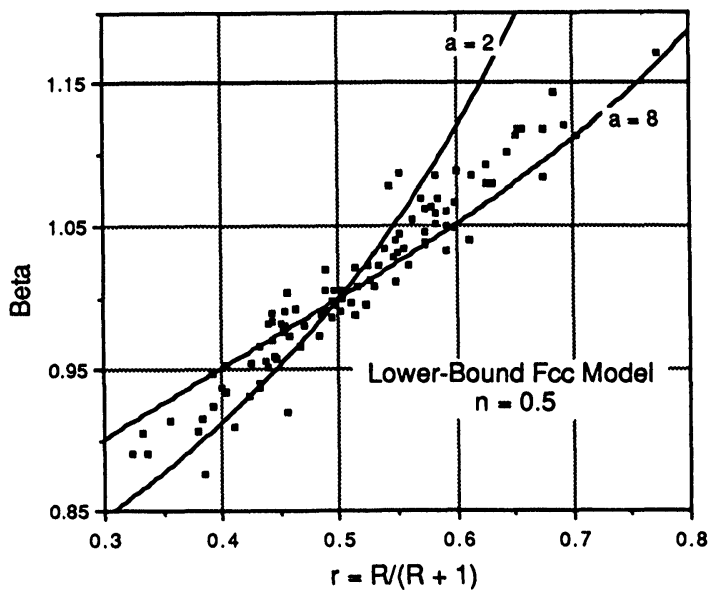

C.

Figure 4 Values of $\beta$ and $r$ for calculated with lower-bound fcc assumptions for various assumed textures, with $n=0.1(\mathrm{~A}), 0.25(\mathrm{~B})$, and $0.5(\mathrm{C})$. The solid lines are the predictions of Eqs. (10) and (12). 


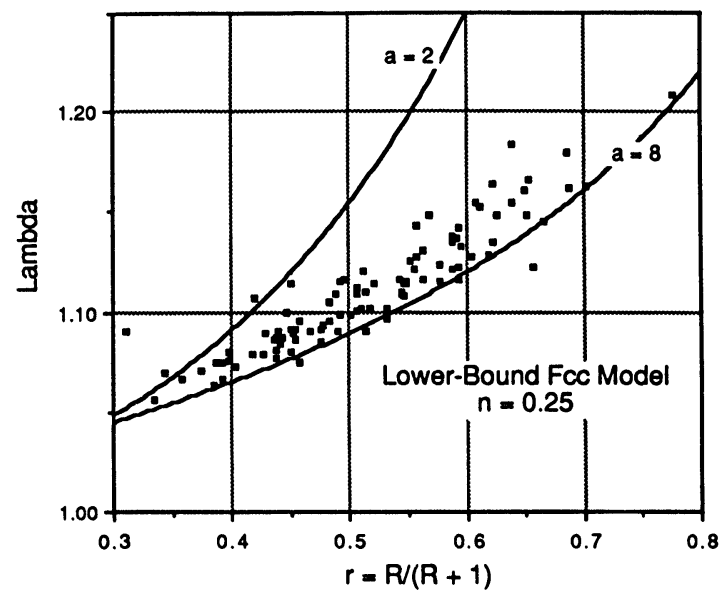

Figure 5 Values of $\lambda$ and $r$ for calculated with lower-bound fcc assumptions for various assumed textures, with $n=0.25$. The solid lines are the predictions of Eqs. (13) and (14).

A more recent criterion (Hosford 1979, Logan and Hosford 1980) has the form

$$
\sigma_{x}^{a}+\sigma_{y}^{a}+R\left(\sigma_{x}-\sigma_{y}\right)^{a}=(R+1) Y^{a}
$$

where $a$ is $a$ positive integer ( $a=6$ and 8 were suggested for bcc and fcc crystals respectively). With this criterion,

$$
\beta=\left\{\left(2+2^{a} R\right) /\left[1+\left(\alpha^{*}\right)^{a}+R\left(1-\alpha^{*}\right)^{a}\right]\right\}^{1 / a},
$$

where $\alpha^{*}$ is the stress ratio for plane strain $\left(\varepsilon_{y}=0\right)$

$$
\alpha^{*}=R^{1 /(a-1)} /\left[1+R^{1 /(a-1)}\right] .
$$

The ratio, $\lambda$, of the strength in plane strain $\left(\varepsilon_{y}=0\right)$ to uniaxial tension is

$$
\lambda=(R+1) / \sqrt{(2 R+1)}
$$

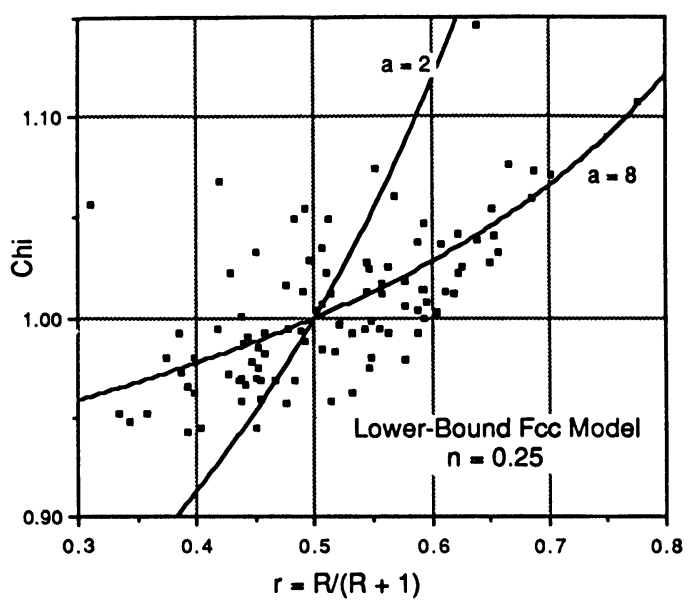

Figure 6 Values of $\chi$ and $r$ for calculated with lower-bound fcc assumptions for various assumed textures, with $n=0.25$. The solid lines are the predictions of Eqs. (15) and (16). 
according to the Hill criterion and

$$
\lambda=\left\{(R+1) /\left[\alpha^{* a}+1+\left(1-\alpha^{*}\right)^{a}\right]\right\}^{1 / a}
$$

according to the new criterion. The biaxial-to-uniaxial strength ratio, $\chi$, is

$$
\chi=[(R+1) / 2]^{1 / 2}
$$

for the Hill criterion and

$$
\chi=[(R+1) / 2]^{1 / a}
$$

for Eq. (11). The lower-bound fcc calculations (with $n=0.25$ ) of $\lambda$ and $\chi$ are compared to the two predictions of the two criteria in Figures 5 and 6.

For the $\langle 111\rangle$ pencil-glide model the plane, slip occurs is the most highly stressed plane containing the active $\langle 111\rangle$ slip direction. Therefore $\cos \phi_{x i}=$ $\sin \lambda_{x i}$ and $m_{x i}=\cos \lambda_{x i} \sin \lambda_{x i}$. Similarly $\cos \phi_{y i}=\sin \lambda_{y i}$ so $m_{y i}=\cos \lambda_{y i} \sin \lambda_{y i}$. In each grain the active slip system is the one for which $\left(m_{x i}+\alpha m_{y i}\right)$ is the highest. The results of calculations for $\langle 111\rangle$ pencil glide were very similar to those for the fcc model.

\section{DISCUSSION OF RESULTS}

In every case the scatter of the calculated results is much better approximated by the new yield criterion (Eq. (11)) with a high exponent than by the Hill criterion (Eq. (9) or Eq. (11) with $a=2$ ). To determine the exponent that gives the best fit, the square of the correlation coeficient, $R^{2}$, between the ratios $(\beta, \lambda$ or $\chi)$ from the lower-bound calculations and the predictions of Eq. (11) for the same values of $r$ were found for various exponents, $a . R^{2}$ was calculated as:

$$
R^{2}=1-\sum\left[X_{\text {cryst }}-X(a)\right] / \sum\left(X_{\text {cryst }}-\bar{X}_{\text {cryst }}\right)
$$

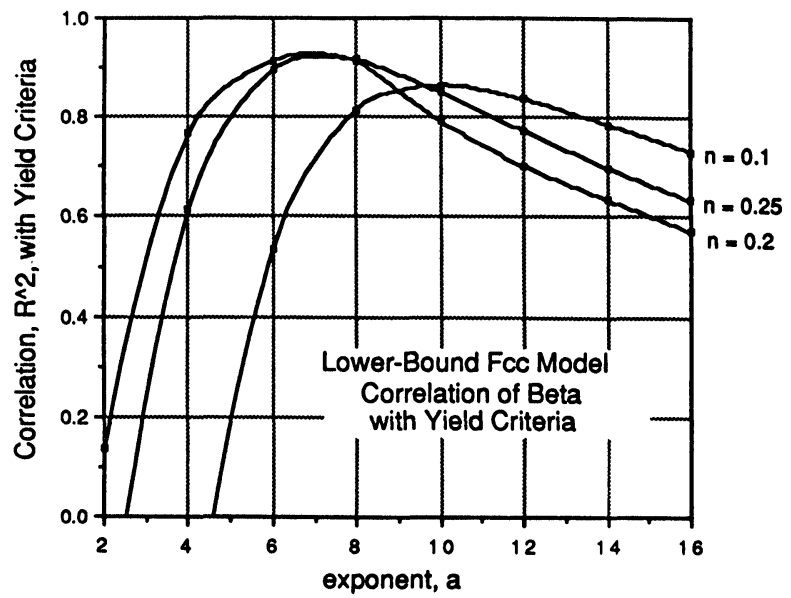

Figure 7 Correlation of the values of $\beta$ calculated using the lower-bound fcc model from values of $\beta$ predicted by Eq. (12) for the same $r$, plotted as a function of the exponent, $a$, in the equation. Curves are shown for the three assumed strain-hardening exponents. 


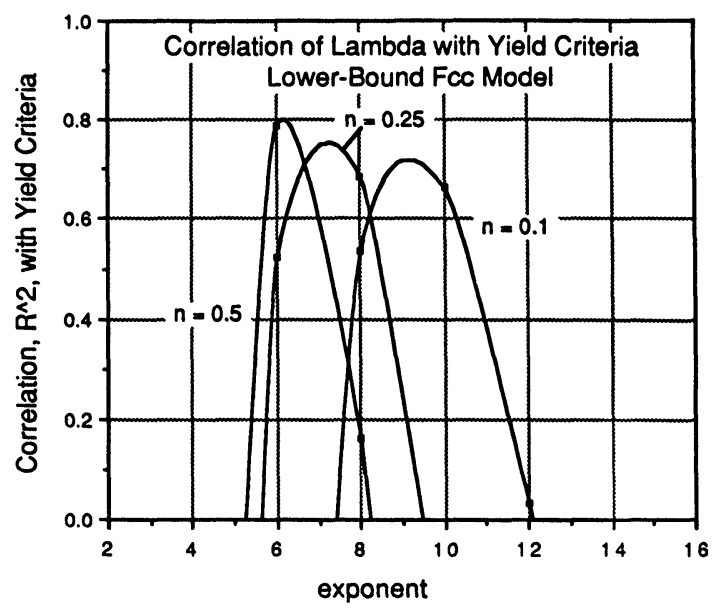

Figure 8 Correlation of the values of $\lambda$ calculated using the lower-bound fcc model from values of $\lambda$ predicted by Eq. (14) for the same $r$, plotted as a function of the exponent, $a$, in the equation. Curves are shown for the three assumed strain-hardening exponents.

where $X_{\text {cryst }}$ is the value of $\beta, \lambda$ or $\chi$ predicted for a texture by the crystallographic model, $X(a)$ is the corresponding value of $\beta, \lambda$ or $\chi$ predicted by Eq. (12), (14) or (16) using the value of $R$ predicted by the crystallographic model for the same texture and $\bar{X}_{\text {cryst }}$ is the average of the calculated values of $X_{\text {cryst }}$. (Note that $R$ in Eq. (17) is different from $R$ in Eq. (9-16)).

The squares of the correlation coefficients are plotted against $a$ in Figures 7 through 12 . In every case $R^{2}$ for $a=2$ was either very low or negative. (A negative value of $R^{2}$ indicates that $X_{\text {cryst }}$ is a better estimate of $X_{\text {cryst }}$ than $X(a)$ is). The results for the fcc and pencil-glide models are very similar. For the $\beta$ and

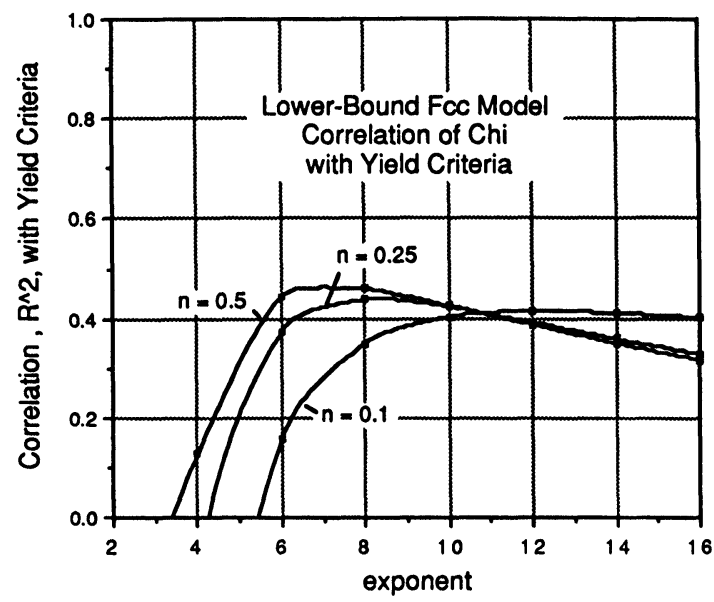

Figure 9 Correlation of the values of $\chi$ calculated using the lower-bound fcc model from values of $\chi$ predicted by Eq. (15) for the same $r$, plotted as a function of the exponent, $a$, in the equation. Curves are shown for the three assumed strain-hardening exponents. 


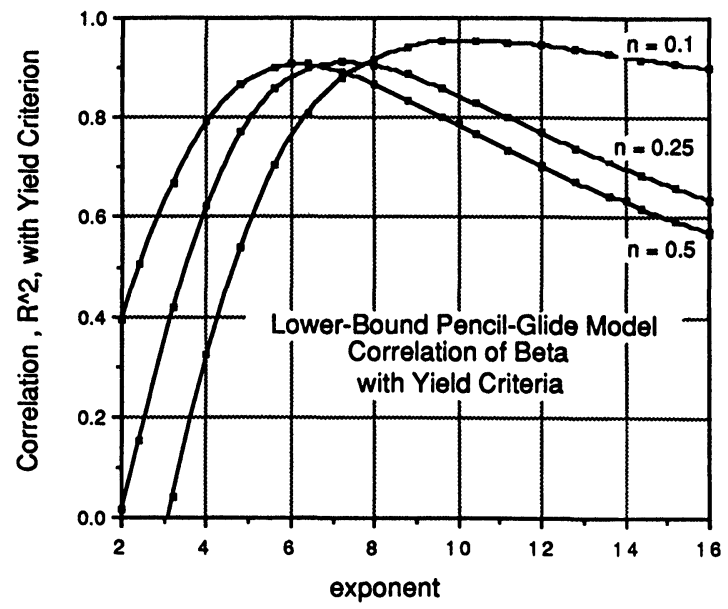

Figure 10 Correlation of the values of $\beta$ calculated using the lower-bound pencil-glide model from values of $\beta$ predicted by Eq. (12) for the same $r$, plotted as a function of the exponent, $a$, in the equation. Curves are shown for the three assumed strain-hardening exponents.

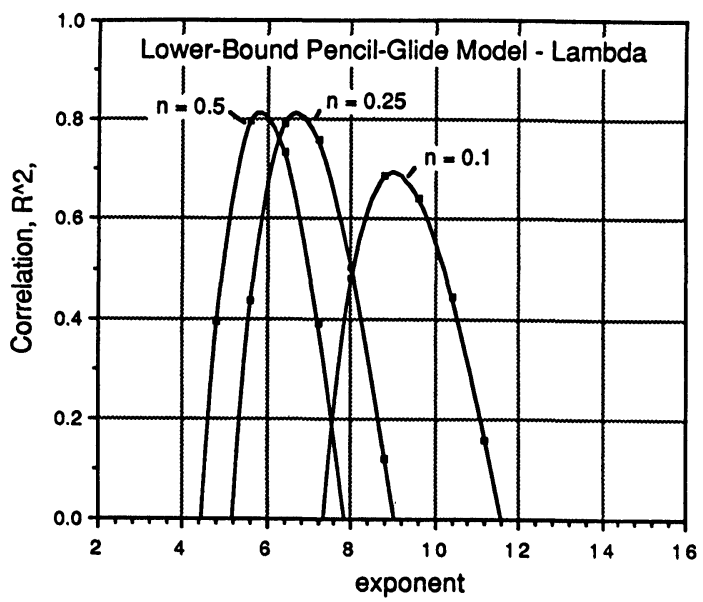

Figure 11 Correlation of the values of $\lambda$ calculated using the lower-bound pencil-glide model from values of $\lambda$ predicted by Eq. (14) for the same $r$, plotted as a function of the exponent, $a$, in the equation. Curves are shown for the three assumed strain-hardening exponents.

$\lambda$ parameters, the best fits occur for exponents between $a=6$ for $n=0.25$ and 0.5 . For $n=0.1$, the best correlation corresponds to about $a=10$. For the biaxial-to-uniaxial strength ratio, chi, the maximum in the $R^{2}$ vs. a curves are not sharp. However it is clear that $6 \leq a \leq 10$ gives a much better fit than $a=2$.

\section{OTHER MODELS}

The term "lower bound" has been incorrectly applied to polycrystalline strength models that do not fulfill the lower-bound requirements of a statically admissable 


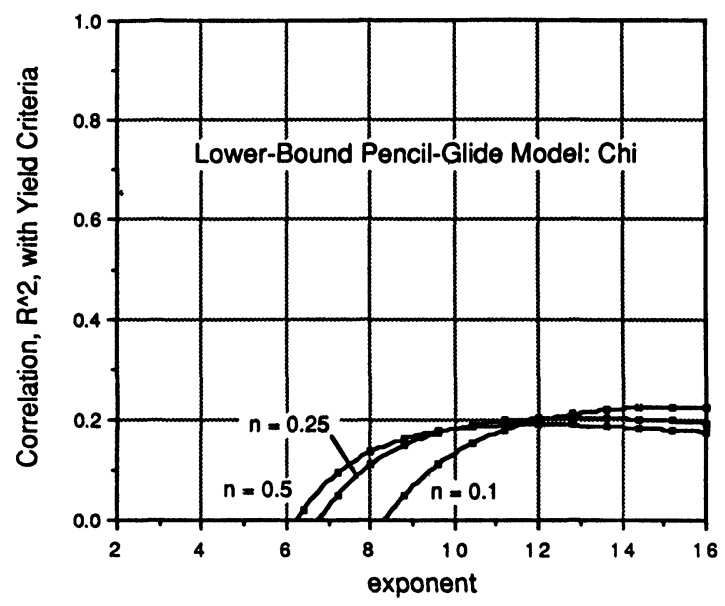

Figure 12 Correlation of the values of $\chi$ calculated using the lower-bound pencil-glide model from values of $\chi$ predicted by Eq. (15) for the same $r$, plotted as a function of the exponent, $a$, in the equation. Curves are shown for the three assumed strain-hardening exponents.

stress field (Piehler 1967, Semiatin et al. 1979, Hosford 1974, Da Viana et al. 1979, Alhoff and Wincierz 1972). Sachs (1928) related the tensile yield strength, $\sigma$, of a randomly oriented polycrystal to the shear stress for slip, $\tau$, by averaging the reciprocal Schmid factors, $1 / m$, and found $\sigma / \tau=(1 / m)_{a v}=2.24$. Although this calculation implies different stresses in each grain, it is a valid lower bound for the special case of a uniaxial tension in a polycrystal having all its grain boundaries parallel to the tensile axis. In this case the stress field is statically admissable. However, when Sachs' model is extended to polycrystals in which the grain boundaries aren't so aligned or to multiaxial loading, it predicts an

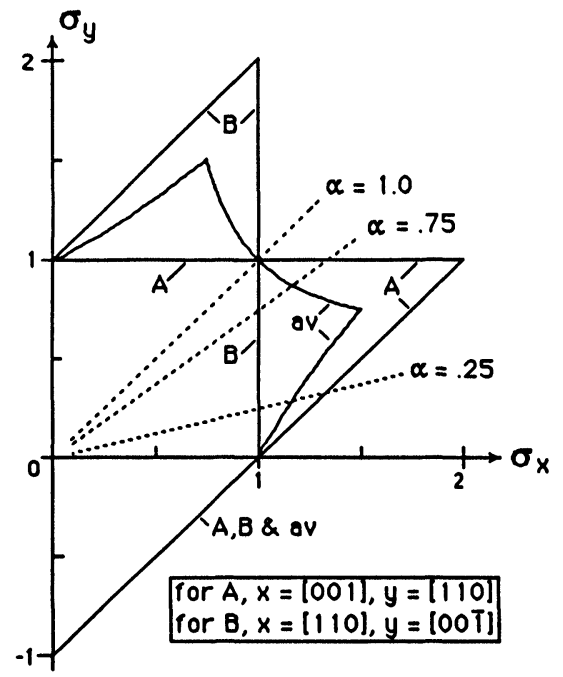

Figure 13 Illustration of how normality may be violated with a model that averages the stresses in grains loaded to yielding under the same ratio of stresses, $\alpha$, (but not the same level of stress). Here, for simplicity only two orientations (A and B) are assumed. The curve found by averaging along lines of equal $\alpha$ is outwardly concave. 
imbalance of forces across grain boundaries and therefore violates the static admissibility requirement of the lower bound theorem.

One way the Sach's model has been extended is to calculate the yield locus by averaging the stresses in grains that are loaded under the same stress ratio, $\alpha$, (but not to the same stress level) (Hill 1948, Piehler and Backofen 1969, Semiatin et al. 1979, Hosford 1974, Da Viana et al. 1979). This is not a lower bound and does not assure normality. An example of how this approach can violate normality follows. Consider the yielding of a polycrystal consisting of two grains: grain $A$ oriented so that $x=[001], y=[110], z=[110]$ and grain $B$ oriented with $x=[110], y=[001], z=[\overline{110}]$. Figure 13 shows the $\sigma_{z}=0$ yield loci for both orientations, as well as the "locus" produced by averaging the two along paths of constant stress ratio. It should be noted that such an averaging scheme results in a locus that is outwardly concave and thus in violation of the normality principle.

\section{CONCLUSIONS}

1) The lower-bound fcc and pencil glide models satisfy normality.

2) The general shape of the yield loci predicted by both lower-bound models are similar and can be fit with yield criterion (Eq. (11)) with a high exponent (6 to 10$)$.

3) The exponent, $a$, giving the best fit increases with the strain hardening exponent, $n$.

4) The extension of the Sach's analysis by assuming that each grain is loaded under the same stress ratio (but not same stress level) violates normality and is not a valid lower bound.

\section{ACKNOWLEDGEMENTS}

The authors wish to acknowledge the support of the AISI during much of the work. Other former students who contributed to the work include R. Logan and S. Argento.

\section{References}

Althoff, J. and Wincerz, P. (1972). The Influence of Texture on the Yield Loci of Copper and Alumimium. Zeitschrift fur Metallkunde, 63, 623-633.

Bishop, J. F. W. and Hill, R. (1951). A Theory of the Plastic Distortion of a Polycrystalline Aggregate under Combined Stress. Philosophical Magazine, 42, 414-427.

Bishop, J. F. W. and Hill, R. (1951). A Theoretical Derivation of the Plastic Properties of a Polycrystalline Face-Centred Metal. Philosophical Magazine, 42, 1298-1307.

Da Viana, C. S., Kallend, J. S. and Davies, G. J. (1979). The Use of Textural Data to Predict the Yield Locus of Metal Sheets. International Journal of Mechanical Sciences, 21, 355-371.

Drucker, D. C., Prager, W. and Greenberg, H. J. (1952). Extended Limit Design Theorems for Continuous Media. Quaterly of Applied Mathematics, 9, 381-389.

Hill, R., (1948). A Theory of Yielding and Plastic Flow of Anisotropic Metals Proceedings of the Royal Society, 193A, 281-297.

Hill, R. (1950). Mathematical Theory of Plasticity, pp. 315-340. London: Oxford University Press. 
Hill, R. (1951). On the State of Stress in a Plastic-Rigid Body at the Yield Point. Philsoophical Magazine, 42, 868-875.

Hosford, W. F. (1974). Yield Locus of Randomly Textured Fcc Metals Calculated with Lower-Bound Assumptions, Metallurgical Transactions, 5, 307-308.

Hosford, W. F. (1979). On Yield Loci of Anisotropic Cubic Metals, 7th North American Metalworking Research Conference Proceedings, SME, Dearborn MI, pp. 191-6.

Hosford, W. F. (1980). Incorporating Work Hardening into Yield Loci Calculations. In Strength of Metals and Alloys (ICSMA 5) edited by P. Haasen, V. Gerold and G. Kostorz, pp., 775-80. Toronto: Pergamon Press.

Logan, R. W. and Hosford, W. F. (1980). Upper-Bound Anisotropic Yield Locus Calculations Assuming $\langle 111\rangle$-Pencil Glide. International Journal of Mechanical Sciences, 22, 419-30.

Piehler, H. R. (1967). Plastic Anisotropy of Body-entered Cubic Metals. Sc.D. Thesis, Massachusetts Institute of Technology, Department of Metallurgy, Cambridge, Massachusetts.

Piehler, H. R. and Backofen, W. A. (1969). The Prediction of Anisotropic Yield for Textured Sheets. In Textures in Research and Practice, edited by J. Grewen and G. Wasserman, pp. 436-43. Berlin: Springer-Verlag.

Sachs, G. (1928). Zietschrift Verein. Deut. Ing., 72, 734-736.

Semiatin, S. L., Morris, P. R. and Piehler, H. R. (1979). Microplasticity Predictions of r-Values and Yield Loci of Low Carbon Steel Sheets Deforming by $\langle 111\rangle$ Pencil Glide. Texture of Crystalline Solids, 3, 191-214.

Taylor, G. I., (1938). Plastic Strain in Metals. Journal of the Institute of Metals, 62, 307-324. 